\title{
ESTABILIDADE DO ÁCIDO ASCÓRBICO E DOS PIGMENTOS DA POLPA DE ACEROLA CONGELADA POR MÉTODOS CONVENCIONAL E CRIOGÊNICO
}

\author{
ANA CAROLINA MOURA DE SENA AQUINO* \\ MARCELO AUGUSTO GUTIERREZ CARNELOSSI** \\ ALESSANDRA ALMEIDA CASTRO***
}

\begin{abstract}
O objetivo deste estudo foi avaliar a estabilidade do ácido ascórbico e dos pigmentos da polpa de acerola, congelada por métodos mecânico (convencional a $-22,1^{\circ} \mathrm{C}$ ) e criogênicos (imersão em vapor de $\mathrm{N}_{2}$ a $-178,6^{\circ} \mathrm{C}$ e em $\mathrm{N}_{2}$ líquido a $-195,1^{\circ} \mathrm{C}$ ), durante armazenamento por 120 dias a $-22,1^{\circ} \mathrm{C}$. Foram determinados os teores de ácido ascórbico, antocianinas totais, carotenoides totais e a cor instrumental da polpa de acerola in natura, pós-congelamento e a cada 30 dias durante os 120 dias de armazenamento. Os teores de ácido ascórbico se mantiveram estáveis para todos os métodos de congelamento, enquanto que a amostra congelada convencionalmente apresentou a maior redução $(16,45 \%)$ nos teores de carotenoides totais em relação ao padrão. Menor diferença de cor $\left(\Delta \mathrm{E}^{*}\right)$ foi verificada para a amostra congelada por vapor de $\mathrm{N}_{2}$, que apresentou redução de $11,26 \%$ e $8,50 \%$ nos teores de carotenoides totais e antocianinas totais, respectivamente, após 120 dias de armazenamento a $-22,1^{\circ} \mathrm{C}$. Diante dos resultados, o congelamento criogênico por vapor de nitrogênio revelou-se boa alternativa para maior retenção dos pigmentos da polpa de acerola.
\end{abstract}

PALAVRAS-CHAVE: ÁCIDO ASCÓRBICO; CONGELAMENTO; CRIOGENIA; PIGMENTOS; POLPA DE ACEROLA.

* Mestre em Ciência e Tecnologia de Alimentos, Núcleo de Pós-Graduação em Ciência e Tecnologia de Alimentos (NUCTA), Universidade Federal de Sergipe (UFS), São Cristóvão, SE (e-mail: carolsena@ ig.com.br).

** Professor Adjunto, Departamento de Tecnologia de Alimentos, UFS, São Cristóvão, SE (e-mail: carnelossi@ ufs.br).

*** Professora Adjunta, Departamento de Tecnologia de Alimentos, UFS, São Cristóvão, SE (e-mail: alessandra@ufs.br). 


\section{INTRODUÇÃO}

Os frutos tropicais apresentam grande potencial econômico e nutricional. A acerola (Malpighia emarginata D.C.) contém alto teor de ácido ascórbico, que como os carotenoides e as antocianinas também presentes nessa fruta são compostos bioativos que se destacam como antioxidantes, elevando esse fruto ao campo dos alimentos funcionais (FREITAS et al., 2006).

A polpa de fruta representa alternativa de consumo para os mercados nacionais ou internacionais, mesmo em períodos de entressafra de produção (LOPES, 2005). No entanto, o processamento afeta o conteúdo, a atividade e a biodisponibilidade dos componentes bioativos dos frutos (MAIA et al., 2007).

A acerola muda de tonalidade com a maturação, passando do verde ao amarelo, laranja, vermelho ou roxo, devido sobretudo à degradação da clorofila e à síntese de antocianinas e carotenoides. Além de pró-vitaminas, os carotenoides são pigmentos responsáveis pela cor de muitas frutas (AGOSTINI-COSTA, ABREU e ROSSETI, 2003). Na acerola, a coloração amarela conferida pelos carotenoides é mascarada pela presença de antocianinas vermelhas que são pigmentos muito instáveis e podem se degradar durante o processamento e a estocagem de alimentos com a consequente alteração da sua cor. Tal mudança afeta a coloração dos produtos fabricados a partir da acerola, principalmente quando se trata de polpas ou sucos (ALVES et al., 2002).

Os pigmentos naturais, tais como antocianinas e carotenoides, contribuem para o aspecto visual dos alimentos, atributo de fundamental importância para sua aceitação e escolha pelos consumidores. Concomitantemente, esses pigmentos apresentam importantes funções e ações biológicas, podendo ser considerados promotores da saúde humana. A associação entre a ingestão de frutas e vegetais e a diminuição do risco de desenvolvimento de diversas desordens crônicodegenerativas já foi reconhecida, e os pigmentos formam um dos grupos de compostos bioativos aos quais são atribuídas tais ações (DE ROSSO, 2006).

A criogenia, de acordo com Vicente, Rubio e Regidor (1994), pode ser definida como a ciência dedicada à produção de baixas temperaturas, sendo o adjetivo criogênico utilizado para denominar gases como nitrogênio que em estado líquido apresentam temperaturas muito baixas $\left(-196^{\circ} \mathrm{C}\right)$. Conforme relatado por Delgado e Rubiolo (2005), o congelamento rápido é vantajoso porque contribui para manter a estrutura celular das polpas congeladas devido à formação de pequenos cristais de gelo.

Diante da escassez de trabalhos com a aplicação do congelamento criogênico associado ao frio mecânico para a conservação de polpas de frutas, o objetivo deste trabalho foi avaliar a estabilidade de polpa de acerola, congelada por diferentes métodos, durante o armazenamento por 120 dias a $-22,1^{\circ} \mathrm{C}$, visando a manutenção dos seus pigmentos e do ácido ascórbico.

\section{MATERIAL E MÉTODOS}

\subsection{AMOSTRAS}

Foram utilizados frutos de acerola in natura, classificados quanto aos estádios de maturação em semi-maduros (mais de $75 \%$ da casca com coloração alaranjada) e maduros (mais de $75 \%$ da casca com coloração vermelha) (CARVALHO e MANICA, 1994), provenientes do Centro de Abastecimento de Aracaju (SE).

Os frutos foram selecionados manualmente para remoção daqueles com injúrias e em estádio de maturação inadequado. As acerolas foram sanitizadas por imersão em solução de hipoclorito de sódio a $30 \mathrm{ppm}$ por 20 minutos, sendo obtida a polpa em despolpadeira da marca Itametal, modelo compacto, utilizando-se tela com 1,5 mm de diâmetro. Acondicionou-se a polpa em sacos de nylon poli 5 camadas, com dimensões de $16 \times 4,5 \mathrm{~cm}$. Após a selagem das embalagens, as amostras de polpa foram submetidas aos seguintes métodos de congelamento: convencional - por frio mecânico (em freezer doméstico vertical a $-22,1^{\circ} \mathrm{C}$ ), criogênico por imersão em vapor de nitrogênio $\left(\mathrm{N}_{2(\mathrm{v})}\right.$, polpas imersas com o auxílio de cânister em botijão criogênico contendo vapor de nitrogênio a $-178,6^{\circ} \mathrm{C}$ ) e criogênico por imersão em nitrogênio líquido $\left(\mathrm{N}_{2(1)}\right.$, imersão das amostras em botijão contendo nitrogênio líquido à temperatura de $-195,1^{\circ} \mathrm{C}$ ). Após criocongeladas, as amostras foram armazenadas em freezer doméstico à temperatura de $-22,1^{\circ} \mathrm{C}$ por 120 dias. 


\subsection{ANÁLISES FÍSICO-QUÍMICAS}

A caracterização da polpa de acerola foi realizada por meio das análises de $\mathrm{pH}$, umidade e acidez total titulável, de acordo com os métodos do IAL (2005). Os sólidos solúveis totais ( ${ }^{\circ}$ Brix) foram determinados mediante leitura em refratômetro Abbé de bancada.

O teor de ácido ascórbico foi determinado pelo método padrão (n. 43.065) da AOAC (1984), modificado por Benassi e Antunes (1988), com a substituição do solvente extrator ácido metafosfórico por ácido oxálico.

Para a quantificação das antocianinas totais, as polpas foram homogeneizadas com solução extratora (etanol $95 \%$ : $\mathrm{HCl} 1,5 \mathrm{~N}-85: 15 \mathrm{v} / \mathrm{v}$ ) e estocadas por 12 horas a $4^{\circ} \mathrm{C}$. Em seguida, foram filtradas e os resíduos lavados exaustivamente com a solução extratora até a remoção completa dos pigmentos. A determinação das antocianinas foi efetuada em triplicata pelo método de $\mathrm{pH}$ diferencial, conforme descrito por Giusti e Wrolstad (2001).

Os carotenoides totais foram avaliados pelo método proposto por Lichtenthaler (1987).

\subsection{COR INSTRUMENTAL}

Para a avaliação da cor instrumental utilizou-se colorímetro Minolta, modelo CR-10, calibrado de fábrica (MINOLTA CORP., 1994), que fornece os parâmetros $L^{*}$ para indicar a luminosidade (claro/escuro); $a^{*}$ para a cromaticidade no eixo da cor verde (-) para vermelha (+); e b* que indica a cromaticidade no eixo da cor azul (-) para amarela $(+)$ do sistema CIELAB. Os parâmetros $C^{*}$ (chroma), $\mathrm{h}$ (ângulo hue) e $\Delta \mathrm{E}^{*}$ (diferença total de cor) foram calculados usando-se as equações 1 , 2 e 3:

$$
\begin{aligned}
& \mathrm{C}^{*}=\left[\left(\mathrm{a}^{*}\right)^{2}+\left(\mathrm{b}^{*}\right)^{2}\right]^{1 / 2} \\
& \mathrm{~h}=\arctan \left(\mathrm{b}^{*} / \mathrm{a}^{*}\right) \\
& \Delta \mathrm{E}^{*}=\left[\left(\Delta \mathrm{L}^{*}\right)^{2}+\left(\Delta \mathrm{a}^{*}\right)^{2}+\left(\Delta \mathrm{b}^{*}\right)^{2}\right]^{1 / 2}
\end{aligned}
$$

Em que:

$\Delta=$ diferença entre cada parâmetro de cor da amostra padrão (in natura) e das amostras armazenadas nos diversos tempos.

Todas as análises foram realizadas em triplicata para a polpa in natura, logo após a aplicação dos diferentes métodos de congelamento, e a cada 30 dias durante 120 dias de armazenamento.

\subsection{ANÁLISE ESTATÍSTICA}

Os dados obtidos foram submetidos à Análise de Variância (ANOVA) e as médias comparadas pelo Teste de Tukey, com $5 \%$ de significância, utilizando-se o programa estatístico Assistat 7.4 beta (SILVA e AZEVEDO, 2006).

\section{RESULTADOS E DISCUSSÃO}

Os tempos médios de congelamento da polpa de acerola por frio mecânico, por $\mathrm{N}_{2(v)}$ e por $\mathrm{N}_{2(1)}$ foram de 3,8 horas, 20 minutos e 3 minutos, respectivamente.

\subsection{CARACTERIZAÇÃO DA POLPA DE ACEROLA}

Na Tabela 1 são apresentados os resultados da caracterização físico-química da polpa de acerola in natura estudada.

O teor de ácido ascórbico da polpa estudada (1946,46 mg de ácido ascórbico.100 g-1) ficou próximo a 1921,00 mg.100 g encontrado por De Rosso e Mercadante (2007), mas superior aos valores apresentados para os frutos de acerola por Vendramini e Trugo (2000), entre 1065,00 e 1074,00 mg.100 g-1, Batista, Figueirêdo e Queiroz (2000) (1650,56 mg.100 g-1) e Yamashita et al. (2003) (1511,00 mg.100 g $\left.\mathrm{g}^{-1}\right)$. 
TABELA 1 - CARACTERIZAÇÃO DA POLPA DE ACEROLA

"IN NATURA"

\begin{tabular}{lc}
\hline Parâmetro & Valor médio \\
\hline $\mathrm{pH}$ & $3,27 \pm 0,01$ \\
Acidez total titulável $\left(\mathrm{g}\right.$ de ácido málico. $\left.100 \mathrm{~g}^{-1}\right)$ & $1,19 \pm 0,04$ \\
Acidez total titulável $\left(\mathrm{g}\right.$ de ácido cítrico. $\left.100 \mathrm{~g}^{-1}\right)$ & $1,03 \pm 0,02$ \\
Sólidos solúveis $\left({ }^{\circ}\right.$ Brix) & $7,42 \pm 0,14$ \\
Umidade $\left(\mathrm{g} .100 \mathrm{~g}^{-1}\right)$ & $91,64 \pm 0,12$ \\
Ácido ascórbico $(\mathrm{mg}$ de ácido ascórbico.100 g-1) & $1946,46 \pm 71,73$ \\
Antocianinas totais $\left(\mathrm{mg}\right.$ de cianidina 3-glicosídeo.100 $\left.\mathrm{g}^{-1}\right)$ & $39,64 \pm 0,96$ \\
Carotenoides totais $\left(\mu \mathrm{gg} .100 \mathrm{~g}^{-1}\right)$ & $960,56 \pm 4,89$ \\
\hline
\end{tabular}

Média \pm desvio padrão (base úmida).

O teor médio de carotenoides totais obtido $\left(960,56 \mu \mathrm{g} .100 \mathrm{~g}^{-1}\right)$ mostrou-se inferior ao apresentado por Mezadri, Pérez-Gálvez e Hornero-Méndez. (2005) que analisando frutos maduros de acerola encontraram 1396,63 $\mu \mathrm{g} .100 \mathrm{~g} \mathrm{~g}^{-1}$. No entanto, está de acordo com os resultados de De Rosso e Mercadante (2005) que ao avaliarem a composição de carotenoides de dois genótipos brasileiros de acerola obtiveram 370,9 e 959,1 $\mu \mathrm{g} .100 \mathrm{~g} \mathrm{~g}^{-1}$ (colheita do ano 2003) e 883,9 e 1881,3 $\mu \mathrm{g} .100 \mathrm{~g}^{-1}$ (colheita do ano 2004).

A concentração de antocianinas totais obtida $(39,64 \mathrm{mg}$ de cianidina 3-glicosídeo.100 $\mathrm{g}^{-1}$ ) está de acordo com os valores apresentados em estudos realizados por Lima et al. (2003) pesquisando polpa congelada proveniente de frutos de 12 diferentes aceroleiras (3,79 a 59,74 mg.100 g-1) e Lima, Mélo e Guerra (2007) que caracterizaram polpas de diferentes genótipos de aceroleiras $\left(6,4\right.$ a $\left.64,6 \mathrm{mg} .100 \mathrm{~g}^{-1}\right)$.

\section{2 ÁCIDO ASCÓRBICO}

De acordo com a Tabela 2, a polpa de acerola congelada mostrou-se estável em relação ao teor de ácido ascórbico para todos os métodos de congelamento, não sendo verificada diferença significativa $(p>0,05)$ durante $o$ armazenamento a $-22,1^{\circ} \mathrm{C}$ por 120 dias. Ao fim do armazenamento foram observadas pequenas reduções de $7,3 \%, 4,5 \%$ e $5,8 \%$ nos teores de ácido ascórbico em relação à polpa no tempo zero para as amostras congeladas pelos métodos convencional, por vapor de $\mathrm{N}_{2}$ e por $\mathrm{N}_{2}$ líquido, respectivamente.

Os teores de ácido ascórbico encontrados enquadraram-se no limite previsto pela Instrução

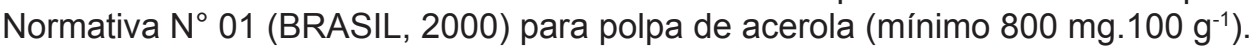

\section{TABELA 2 - VALORES DE ÁCIDO ASCÓRBICO (mg.100 $\left.\mathrm{g}^{-1}\right)$ DAS POLPAS DE ACEROLA SUBMETIDAS A DIFERENTES MÉTODOS DE CONGELAMENTO E ARMAZENADAS POR 120 DIAS A $-22,1^{\circ} \mathrm{C}$}

\begin{tabular}{|c|c|c|c|c|c|}
\hline \multirow{2}{*}{$\begin{array}{l}\text { Métodos de } \\
\text { congelamento }\end{array}$} & \multicolumn{5}{|c|}{ Tempo de armazenamento (dias) } \\
\hline & 0 & 30 & 60 & 90 & 120 \\
\hline polpa in natura & $1946,46^{\mathrm{a}} \pm 71,73$ & - & - & - & - \\
\hline convencional & $1894,37^{\mathrm{aA}} \pm 74,57$ & $1878,57^{\mathrm{aA}} \pm 81,34$ & $1839,94^{\mathrm{aA}} \pm 77,73$ & $1807,00^{\mathrm{aA}} \pm 71,80$ & $1803,96^{\mathrm{aA}} \pm 71,01$ \\
\hline vapor de $\mathrm{N}_{2}$ & $1892,29{ }^{\mathrm{aA}} \pm 74,49$ & $1918,44^{\mathrm{aA}} \pm 79,04$ & $1881,62{ }^{\mathrm{aA}} \pm 71,81$ & $1863,01^{\mathrm{aA}} \pm 72,34$ & $1858,08^{\mathrm{aA}} \pm 69,96$ \\
\hline $\mathrm{N}_{2}$ líquido & $1895,04{ }^{\mathrm{aA}} \pm 74,60$ & $1877,63^{\mathrm{aA}} \pm 81,30$ & $1790,06^{\mathrm{aA}} \pm 27,51$ & $1858,30^{\mathrm{aA}} \pm 57,98$ & $1832,68^{\mathrm{aA}} \pm 68,00$ \\
\hline
\end{tabular}

Média \pm desvio padrão.

Letras minúsculas comparam médias na mesma coluna entre os diferentes tratamentos de congelamento no mesmo tempo e maiúsculas comparam médias na mesma linha para o mesmo tratamento de congelamento em diferentes tempos. Letras iguais não diferem significativamente de acordo com o teste de Tukey, ao nível de $5 \%$ de probabilidade. 
Oliveira et al. (2001) avaliaram a estabilidade da polpa de acerola branqueada e estocada sob temperatura de congelamento e também não verificaram variação significativa no teor de vitamina C $\left(1319,00 \pm 58,00 \mathrm{mg} .100 \mathrm{~g}^{-1}\right)$ ao longo de 12 meses.

Yamashita et al. (2003), estudando a estabilidade da vitamina $\mathrm{C}$ em produtos de acerola, constataram perda de aproximadamente $3 \%$ após 4 meses de armazenagem a $-12^{\circ} \mathrm{C}$ e $-18^{\circ} \mathrm{C}$. Pequena redução também foi observada por Araújo et al. (2007) quando avaliaram as alterações de ácido ascórbico na polpa de frutos de clones de aceroleira conservada por congelamento $\left(-18^{\circ} \mathrm{C}\right)$ durante 12 meses. Os autores justificaram esse comportamento pela alta acidez da polpa, que auxilia na manutenção desse nutriente.

\subsection{CAROTENOIDES TOTAIS}

Após 120 dias de armazenamento a $-22,1^{\circ} \mathrm{C}$, todos os métodos de congelamento promoveram redução significativa $(p \leq 0,05)$ nos teores de carotenoides totais das amostras de polpa de acerola em relação ao tempo zero (Tabela 3).

\section{TABELA 3 - VALORES DE CAROTENOIDES TOTAIS $\left(\mu \mathrm{g} .100 \mathrm{~g}^{-1}\right)$ DAS POLPAS DE ACEROLA SUBMETIDAS A DIFERENTES MÉTODOS DE CONGELAMENTO E ARMAZENADAS POR 120 DIAS A $-22,1^{\circ} \mathrm{C}$}

\begin{tabular}{lccccc}
\hline \multirow{2}{*}{$\begin{array}{c}\text { Métodos de } \\
\text { congelamento }\end{array}$} & $\mathbf{0}$ & $\mathbf{3 0}$ & $\mathbf{6 0}$ & $\mathbf{9 0}$ & $\mathbf{1 2 0}$ \\
\cline { 2 - 6 } polpa in natura & $960,56^{\mathrm{a}} \pm 4,89$ & - & - & - & - \\
convencional & $917,10^{\mathrm{CA}} \pm 5,92$ & $904,99^{\mathrm{bB}} \pm 2,48$ & $868,98^{\mathrm{cC}} \pm 4,22$ & $802,59^{\mathrm{cC}} \pm 2,65$ & $807,41^{\mathrm{cC}} \pm 5,49$ \\
vapor de $\mathrm{N}_{2}$ & $948,85^{\mathrm{bA}} \pm 2,52$ & $939,59^{\mathrm{aA}} \pm 5,37$ & $906,36^{\mathrm{bB}} \pm 6,42$ & $850,32^{\mathrm{bC}} \pm 3,66$ & $852,42^{\mathrm{bC}} \pm 1,74$ \\
$\mathrm{~N}_{2}$ líquido & $950,08^{\mathrm{bA}} \pm 3,86$ & $948,21^{\mathrm{aA}} \pm 2,67$ & $932,82^{\mathrm{aB}} \pm 4,12$ & $897,15^{\mathrm{aC}} \pm 2,16$ & $896,70^{\mathrm{aC}} \pm 3,66$ \\
\hline
\end{tabular}

Média \pm desvio padrão.

Letras minúsculas comparam médias na mesma coluna entre os diferentes tratamentos de congelamento no mesmo tempo e maiúsculas comparam médias na mesma linha para o mesmo tratamento de congelamento em diferentes tempos. Letras iguais não diferem significativamente de acordo com o teste de Tukey, ao nível de $5 \%$ de probabilidade.

Pequenas reduções de carotenoides $(1,09$ a $4,52 \%)$, no entanto significativas $(p \leq 0,05)$, foram observadas nas amostras logo após os tratamentos de congelamento. Após 90 dias de armazenamento a $-22,1^{\circ} \mathrm{C}$, as amostras congeladas convencionalmente, por $\mathrm{N}_{2(\mathrm{v})}$, e por $\mathrm{N}_{2(1)}$ apresentaram reduções de carotenoides totais de 16,45\%,11,48\% e 6,60\%, respectivamente.

$\mathrm{Na}$ acerola, a coloração amarela conferida pelos carotenoides é mascarada pela presença de antocianinas vermelhas. De acordo com Agostini-Costa, Abreu e Rosseti (2003), a polpa congelada não branqueada de acerola estocada apresenta descoloração esporádica, tornando-se amarela e acarretando prejuízos para sua comercialização.

Perdas consideráveis de carotenoides ocorreram em polpas de acerola não branqueadas, estocadas por 120 dias a $-22,1^{\circ} \mathrm{C}$, indicando que houve oxidação enzimática (CAVALCANTE e RODRIGUEZ-AMAYA, 1995). Nesse estudo, a maior redução foi observada para a amostra congelada convencionalmente e a menor redução para aquela criocongelada por imersão em $\mathrm{N}_{2(1)}$.

\subsection{ANTOCIANINAS TOTAIS}

O teor de antocianinas totais das polpas de acerola decresceu, significativamente $(p \leq 0,05)$, logo após os congelamentos convencional a $-22,1^{\circ} \mathrm{C}$ e por imersão em $\mathrm{N}_{2(1)}$ a $-195,1^{\circ} \mathrm{C}$ com reduções médias de $7,06 \%$ e $24,22 \%$, respectivamente, em relação a polpa in natura (Tabela 4).

A amostra congelada pelo método convencional, após os 120 dias de armazenamento, apresentou redução percentual de $21,67 \%$ no teor de antocianinas totais. Lopes (2005), estudando a estabilidade da polpa de acerola sob as mesmas condições de congelamento, observou diminuição de $18,02 \%$ nas antocianinas totais após 120 dias de estocagem. Silva, Menezes e Guedes (1999) armazenaram polpa de acerola sem tratamento térmico, sob congelamento por seis meses e verificaram $21,74 \%$ de redução de antocianinas. 


\section{TABELA 4 - VALORES DE ANTOCIANINAS TOTAIS (mg DE CIANIDINA 3-GLICOSÍDEO.100g-1) DAS POLPAS DE ACEROLA SUBMETIDAS A DIFERENTES MÉTODOS DE CONGELAMENTO E ARMAZENADAS POR 120 DIAS A $-22,1^{\circ} \mathrm{C}$}

\begin{tabular}{|c|c|c|c|c|c|}
\hline \multirow{2}{*}{$\begin{array}{c}\text { Métodos de } \\
\text { congelamento }\end{array}$} & \multicolumn{5}{|c|}{ Tempo de armazenamento (dias) } \\
\hline & 0 & 30 & 60 & 90 & 120 \\
\hline polpa in natura & $39,64^{a} \pm 0,96$ & - & - & - & - \\
\hline convencional & $36,84^{\mathrm{bA}} \pm 0,99$ & $34,61^{\mathrm{bAB}} \pm 0,89$ & $32,28{ }^{a B} \pm 0,96$ & $31,72^{a b B C} \pm 1,39$ & $31,05^{b c} \pm 0,67$ \\
\hline vapor de $\mathrm{N}_{2}$ & $39,52^{\mathrm{aA}} \pm 0,96$ & $38,41^{\mathrm{aAB}} \pm 1,67$ & $37,85^{\mathrm{aAB}} \pm 2,55$ & $36,84^{\mathrm{aB}} \pm 3,86$ & $36,27^{\mathrm{aB}} \pm 1,93$ \\
\hline $\mathrm{N}_{2}$ líquido & $30,04^{\mathrm{CA}} \pm 1,67$ & $29,48^{\mathrm{cA}} \pm 0,97$ & $28,37^{\mathrm{bA}} \pm 2,89$ & $28,93^{\mathrm{bA}} \pm 1,55$ & $28,36^{\mathrm{bA}} \pm 0,96$ \\
\hline
\end{tabular}

Média \pm desvio padrão.

Letras minúsculas comparam médias na mesma coluna entre os diferentes tratamentos de congelamento no mesmo tempo e maiúsculas comparam médias na mesma linha para o mesmo tratamento de congelamento em diferentes tempos. Letras iguais não diferem significativamente de acordo com o teste de Tukey, ao nível de $5 \%$ de probabilidade.

Lima et al. (2003), avaliando o teor de antocianinas em polpa de acerola congelada proveniente de frutos de doze diferentes aceroleiras, constataram reduções de 3,4 a $23,6 \%$ nos teores de antocianinas totais após 6 meses de armazenamento a $-18^{\circ} \mathrm{C}$. Justificaram essa variação na redução do teor desse constituinte entre as diferentes amostras pelas características intrínsecas dos frutos de cada planta.

As reações metabólicas são reduzidas durante o congelamento, porém não totalmente inibidas, o que justifica a degradação dos pigmentos antociânicos nas polpas armazenadas a $-18^{\circ} \mathrm{C}$ (CHEFTEL, CHEFTEL e BESANÇON, 1983). De acordo com Araújo et al. (2007) as antocianinas são responsáveis pela coloração vermelha da acerola, sendo importante mensurá-las devido ao interesse comercial estar voltado para a aparência. Assim, a polpa de acerola amarelada será provavelmente recusada pelos consumidores.

A polpa congelada por $\mathrm{N}_{2(\mathrm{v})}$ evidenciou a maior retenção dos teores de antocianinas $(91,50 \%)$ até o final do experimento, enquanto que a amostra congelada em $\mathrm{N}_{2(1)}$ revelou a menor retenção desses pigmentos $(71,54 \%)$. Tais alterações no teor de antocianinas totais também foram observadas visualmente, mediante certa descoloração das polpas congeladas convencionalmente e por imersão em $\mathrm{N}_{2(1)}$. Isso se deve, possivelmente, pelo contato direto das antocianinas (que não estavam mais intactas nas células devido ao processamento dos frutos para obtenção da polpa), com o nitrogênio líquido, podendo a redução brusca de temperatura ter provocado a deterioração desses pigmentos.

As variações nos teores de antocianinas totais ao longo do período de armazenamento poderiam ser justificadas pela interconversão das quatro formas estruturais de antocianinas (base quinoidal, cátion flavilium, pseudobase ou carbinol e chalcona) que em solução aquosa ácida se encontram em equilíbrio (MAZZA e BROUILLARD, 1987). A degradação das antocianinas também pode ter sido favorecida por ação enzimática, já que a polpa em estudo não foi submetida à nenhum tratamento térmico. As glicosidases, também denominadas de antocianidases, hidrolisam as antocianinas liberando os açúcares e as antocianidinas que são mais instáveis do que as antocianinas (FRANCIS, 1989).

\subsection{COR INSTRUMENTAL}

Os valores dos parâmetros de cor da polpa in natura foram: $a^{*} 27,00 ; b^{*} 32,00 ; L^{*} 32,00$; $C^{*} 41,87$ e h 49,84 . De acordo com a Figura 1 houve decréscimo nos parâmetros $a^{*}, b^{*}$ e $C^{*} e$ aumento de $\mathrm{L}^{*} \mathrm{e} \mathrm{h}$ das polpas de acerola ao longo do tempo de armazenamento.

Foram verificadas alterações médias nos valores de $a^{*}(24,81 \%, 20,37 \%$ e $31,11 \%)$, de b* $(18,13 \%, 14,06 \%$ e $9,69 \%)$ e L* $(9,38 \%, 8,75 \%$ e 9,38\%) em relação ao tempo zero, após 120 dias de armazenamento para as amostras congeladas convencionalmente, por $\mathrm{N}_{2(v)}$ e por $\mathrm{N}_{2(l)}$, respectivamente. 
A polpa congelada por imersão em $\mathrm{N}_{2(1)}$, após 120 dias de armazenamento a $-22,1^{\circ} \mathrm{C}$ apresentou o menor valor de vermelho $\left(a^{*}=18,60\right)$ e os maiores valores de luminosidade $\left(L^{*}=35,00\right)$, de amarelo $\left(b^{*}=28,90\right)$, de cromaticidade $\left(C^{*}=34,37\right)$ e de ângulo hue $(h=57,23)$, caracterizando amostra de cor clara, amarelada e intensa.
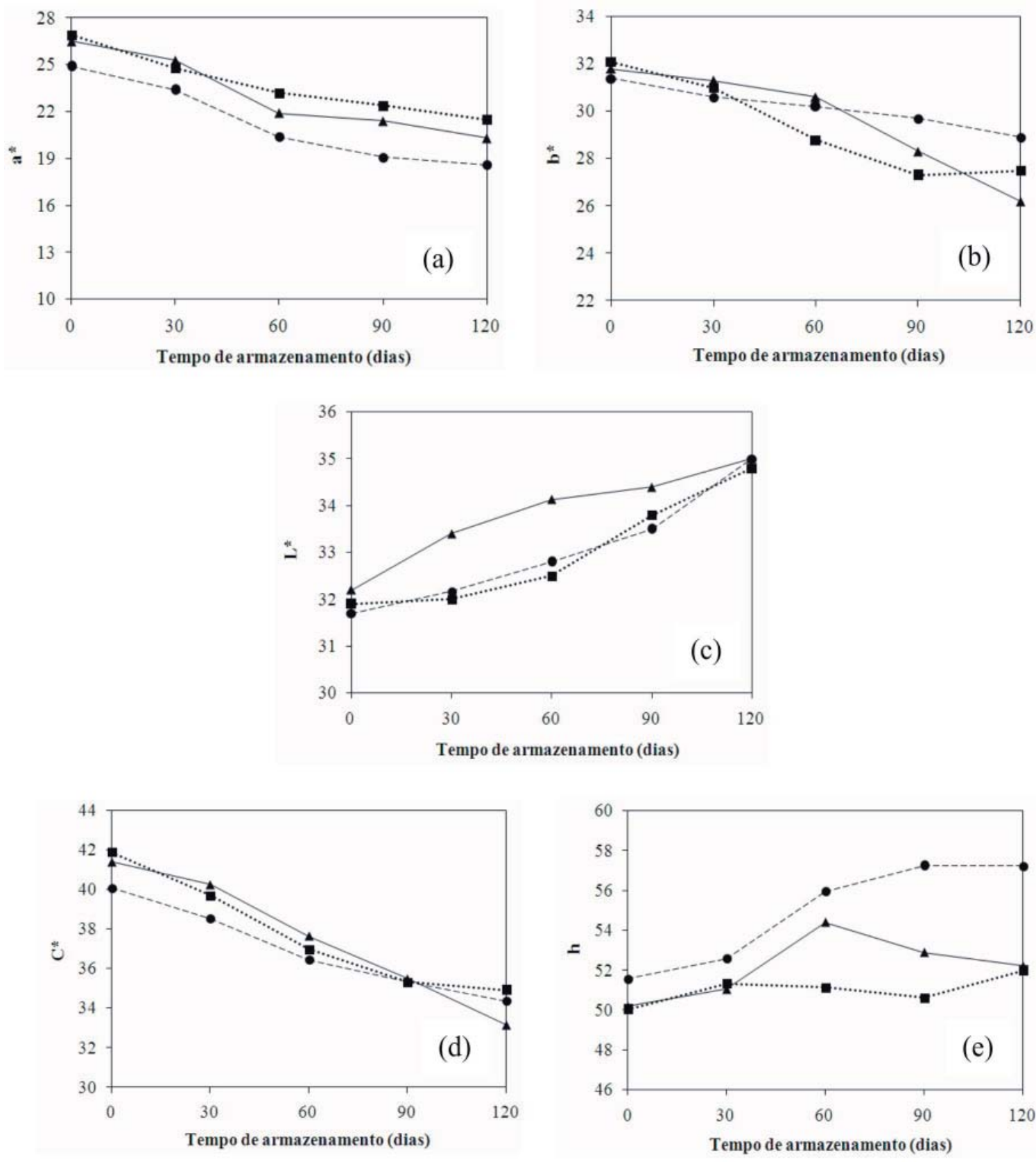

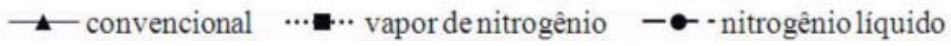

FIGURA 1 - ALTERAÇÕES NOS PARÂMETROS DE COR $a^{*}(a), b^{*}(b), L^{*}(c), C^{*}(d)$ E h (e) DAS POLPAS DE ACEROLA SUBMETIDAS A DIFERENTES MÉTODOS DE CONGELAMENTO E ARMAZENADAS POR 120 DIAS A $-22,1^{\circ} \mathrm{C}$

Em decorrência da degradação das antocianinas, os valores de $a^{*}$ decresceram durante o armazenamento e como consequência os valores de $L^{*}$ aumentaram. Já o parâmetro $C^{*}$, relacionado com a intensidade de cor, diminuiu. A perda da cor vermelha foi evidenciada pelo aumento do ângulo hue (h), indicando a mudança da cor vermelha para laranja. Dessa forma, a amostra congelada por imersão em $\mathrm{N}_{2(1)}$ apresentou coloração mais próxima a amarela $\left(90^{\circ}\right)$. 
De acordo com a Tabela 5, houve aumento estatisticamente significativo $(p \leq 0,05)$ da diferença total de cor $\left(\Delta \mathrm{E}^{*}\right)$ da polpa de acerola para todas as técnicas de congelamento com o tempo de armazenamento. Verificou-se que a amostra congelada por $\mathrm{N}_{2(\mathrm{~V})}$ obteve os menores valores de $\Delta \mathrm{E}^{*}$, em relação a amostra padrão (in natura), ou seja, menor alteração na cor.

\section{TABELA 5 - DIFERENÇA TOTAL DE COR ( $\left.\triangle E^{*}\right)$ EM RELAÇÃO AO PADRÃO DAS POLPAS DE ACEROLA CONGELADAS POR DIFERENTES MÉTODOS EM FUNÇÃO DO TEMPO DE ARMAZENAMENTO A $-22,1^{\circ} \mathrm{C}$}

\begin{tabular}{cccc}
\hline \multirow{2}{*}{$\begin{array}{c}\text { Tempo de armazenamento } \\
\text { (dias) }\end{array}$} & \multicolumn{3}{c}{ Métodos de congelamento } \\
\cline { 2 - 4 } & convencional & vapor de $\mathbf{N}_{\mathbf{2}}$ & $\mathbf{N}_{\mathbf{2}}$ líquido \\
\hline 0 & $0,57 \pm 0,06^{\mathrm{a}}$ & $0,17 \pm 0,04^{\mathrm{a}}$ & $2,20 \pm 0,04^{\mathrm{a}}$ \\
30 & $2,31 \pm 0,03^{\mathrm{b}}$ & $2,42 \pm 0,08^{\mathrm{b}}$ & $3,87 \pm 0,06^{\mathrm{b}}$ \\
60 & $5,70 \pm 0,02^{\mathrm{c}}$ & $4,99 \pm 0,06^{\mathrm{c}}$ & $6,89 \pm 0,04^{\mathrm{c}}$ \\
90 & $7,13 \pm 0,06^{\mathrm{d}}$ & $6,82 \pm 0,07^{\mathrm{d}}$ & $8,36 \pm 0,08^{\mathrm{d}}$ \\
120 & $9,36 \pm 0,05^{\mathrm{e}}$ & $7,64 \pm 0,04^{\mathrm{e}}$ & $9,44 \pm 0,05^{\mathrm{e}}$ \\
\hline
\end{tabular}

Média \pm desvio padrão.

Médias com expoentes diferentes na mesma coluna indicam diferença estatística $(p \leq 0,05)$.

\section{CONCLUSÃO}

As polpas de acerola congeladas pelos diferentes métodos mantiveram sua concentração de ácido ascórbico estável durante os 120 dias de armazenamento a $-22,1^{\circ} \mathrm{C}$.

O método criogênico de imersão em vapor de nitrogênio proporcionou menor alteração de cor na polpa de acerola, apresentando menores reduções nos teores de carotenoides totais e antocianinas totais após 120 dias de armazenamento a $-22,1^{\circ} \mathrm{C}$.

Conclui-se que o método criogênico por imersão em vapor de nitrogênio representa boa alternativa para o congelamento da polpa de acerola, devido melhor manutenção dos seus pigmentos e menor redução das alterações da cor do produto, comumente, observadas quando se usa o método mecânico.

\section{ABSTRACT}

\section{STABILITY OF ASCORBIC ACID AND PIGMENTS OF FROZEN ACEROLA PULP USING CONVENTIONAL AND CRYOGENIC PROCESSES}

The objective of this study was to evaluate the stability of ascorbic acid and pigments of frozen acerola pulp using mechanical (conventional at $-22.1^{\circ} \mathrm{C}$ ) and cryogenics (immersion in steam of $\mathrm{N}_{2}$ at $-178.6^{\circ} \mathrm{C}$ and immersion in liquid $\mathrm{N}_{2}$ to $-195.1^{\circ} \mathrm{C}$ ) processes during 120 days storage at $-22.1^{\circ} \mathrm{C}$. Were determined the ascorbic acid content, total anthocyanins and carotenoids as well as instrumental color of the acerola pulp in natura, postfreeze and at every 30 days during the storage period. The levels of ascorbic acid were stable at all freezing methods, while the conventionally frozen sample presented the greatest reduction $(16.45 \%)$ on carotenoids levels in comparison to the standard sample. The smaller difference in color $\left(\Delta \mathrm{E}^{*}\right)$ was observed for the sample frozen by immersion in steam of $\mathrm{N}_{2}$, which demonstrated a reduction of $11.26 \%$ and $8.50 \%$ in the levels of carotenoids and anthocyanins, respectively, after 120 days storage at $-22.1^{\circ} \mathrm{C}$. According to the results, the cryogenic freezing in nitrogen vapor before the conventional process is a good alternative to higher retention of the pigments of the pulp.

KEY-WORDS: ASCORBIC ACID; FREEZING; CRYOGENIC; PIGMENTS; ACEROLA PULP.

\section{REFERÊNCIAS}

1 AGOSTINI-COSTA, T.S.; ABREU, L.N.; ROSSETI, A.G. Efeito do congelamento e do tempo de estocagem da polpa de acerola sobre o teor de carotenóides. Revista Brasileira de Fruticultura, v. 25, n. 1, p. 56-58, 2003. 
2 ALVES, R.E.; FILGUEIRAS, H.A.C.; MOSCA, J.L.; CHITARRA, A.B.; MENEZES, J.B. Qualidade de acerolas congeladas e submetidas a aplicação pós-colheita de cálcio e aditivos. Interamerican Society for Tropical Horticulture, v. 46, p. 4-7, Out. 2002

3 AOAC. Association of Official Analytical Chemists. Official methods of analysis of AOAC International. $14^{\text {th }}$ ed. Arlington, 1984.

4 ARAÚJO, P.G.L.; FIGUEIREDO, R.W.; ALVES, R.E.; MAIA, G.A.; PAIVA, J.R. $\beta$-caroteno, ácido ascórbico e antocianinas totais em polpa de frutos de aceroleira conservada por congelamento durante 12 meses. Ciência e Tecnologia de Alimentos, v. 27, n.1, p. 104-107, 2007.

5 BATISTA, M.S.; FIGUEIRÊDO, R.M.F.; QUEIROZ, A.M. Parâmetros físicos e químicos da acerola (Malpighia punicifolia, L.) em diferentes fases de maturação. Revista Brasileira de Produtos Agroindustriais, v.2, n.2, p.19-24, 2000.

6 BENASSI, M.T.; ANTUNES, A.J. A comparison of metaphosphoric and oxalic acids as extractants solutions for the determination of vitamin $C$ in selected vegetables. Arquivos de Biologia e Tecnologia, v. 31, n. 4, p. 507-513, 1988.

7 BRASIL. Ministério da Agricultura e do Abastecimento. Instrução Normativa n. 01, de 07/01/2000. Regulamento técnico geral para fixação dos padrões de identidade e qualidade para polpa de fruta. Diário Oficial [da] República Federativa do Brasil, Brasília. Disponível em: <http://www.agricultura.gov.br>. Acesso em: 25 de outubro de 2008.

8 CARVALHO, R.I.N.; MANICA, I. Influência de estádios de maturação e condições de armazenamento na conservação da acerola (Malpighia glabra L.). Pesquisa Agropecuária Brasileira, v. 29, n. 5, p. 681-688, 1994.

9 CAVALCANTE, M. L.; RODRIGUEZ-AMAYA, D. B. Alteration of the carotenoid composition during manufacture and storage of frozen Eugenia uniflora fruit. In: WORLD CONGRESS OF FOOD SCIENCE AND TECHNOLOGY, $9^{\text {th }}$, Budapeste HUN. Abstracts...Budapeste: IUFOST, 1995. p.32.

10 CHEFTEL, J.C.; CHEFTEL, H.; BESANÇON, P. Métodos de conservacíon. In: CHEFTEL, J.C.; CHEFTEL, H.; BESANÇON, P. (Ed.) Introduccion a la bioquímica y tecnología de los alimentos. Zaragoza: Acribia, 1983. v. 2, p.173-202.

11 DE ROSSO, V. V. Composição de carotenóides e antocianinas em acerola. Estabilidade e atividade antioxidante em sistemas-modelo de extratos antociânicos de acerola e açaí. 2006. 154 p. Tese (Doutorado em Ciência de Alimentos) - Faculdade de Engenharia de Alimentos, Universidade Estadual de Campinas, Campinas, 2006.

12 DE ROSSO, V.V.; MERCADANTE, A.Z. Carotenoid composition of two Brazilian genotypes of acerola (Malpighia punicifolia L.) from two harvests. Food Research International, v. 38, p. 1073-1077, 2005.

13 DE ROSSO, V.V.; MERCADANTE, A.Z. The high ascorbic acid content is the main cause of the low stability of anthocyanin extracts from acerola. Food Chemistry, v. 103, p. 935-943, 2007.

14 DELGADO, A.E.; RUBIOLO, A.C. Microstructural changes in strawberry after freezing and thawing processes. Lebensmittel-Wissenschaft und-Technologie, v. 38, n. 2, p. 135-142, 2005.

15 FRANCIS, F.J. Food colorants anthocyanins. CRC Critical Review in Food Science and Nutrition, v. 28, n. 4, p. 273$314,1989$.

16 FREITAS, C.A.S.; MAIA, G.A.; COSTA, J.M.C.; FIGUEIREDO, R.W.; SOUZA, P.H.M. Acerola: produção, composição, aspectos nutricionais e produtos. Revista Brasileira de Agrociência, v. 12, n. 4, p. 395-400, out.dez. 2006.

17 GIUSTI, M.M.; WROLSTAD, R.E. Characterization and measurement of anthocyanins by UV-visible spectroscopy. In: WROLSTAD, R.E. (Ed.). Current protocols in food analytical chemistry. New York: John Wiley \& Sons, 2001.

18 IAL. Instituto Adolfo Lutz. Métodos físico-químicos para análises de alimentos. 4. ed. São Paulo, 2005. 1018 p.

19 LICHTENTHALER, H.K. Chlorophylls and carotenoids: pigments of photosynthetic biomembranes. In: PACKER, L.; DOUCE, R. (Eds.). Methods in enzimology. London: Academic Press, 1987. v.148, p.350-81.

20 LIMA, V.L.A.G.; MÉLO, E.A.; GUERRA, N.B. Correlação entre o teor de antocianinas e caracterização cromática de polpas de diferentes genótipos de aceroleira. Brazilian Journal of Food Technology, v. 10, n. 1, p. 51-55, 2007.

21 LIMA, V.L.A.G.; MÉLO, E.A.; MACIEL, M.I.S.; LIMA, D.E.S. Avaliação do teor de antocianinas em polpa de acerola congelada proveniente de frutos de 12 diferentes aceroleiras (Malpighia emarginata D.C.). Ciência e Tecnologia de Alimentos, v. 23, n. 1, p. 101-103, 2003.

22 LOPES, A.S. Pitanga e acerola: estudo de processamento, estabilidade e formulação de néctar misto. 2005. 193 p. Tese (Doutorado em Tecnologia de Alimentos) - Faculdade de Engenharia de Alimentos, Universidade Estadual de Campinas, Campinas, 2005.

23 MAIA, G.A.; SOUSA, P.H.M.; SANTOS, G.M. et al. Efeito do processamento sobre componentes do suco de acerola. Ciência e Tecnologia de Alimentos, v. 27, n.1, p.130-134, 2007. 
24 MAZZA, G.; BROUILLARD, R. Recent developments in the stabilization of anthocyanins in food products. Food Chemistry, v. 25, p. 207-225, 1987.

25 MEZADRI, T.; PÉREZ-GÁLVEZ, A.; HORNERO-MÉNDEZ, D. Carotenoid pigments in acerola fruits (Malpighia emarginata DC.) and derived products. European Food Research and Technology, v. 220, p. 63-69, 2005.

26 MINOLTA CORP. Precise color communication: color control from feeling to instrumentation. Ramsey, 1994. $49 \mathrm{p}$.

27 OLIVEIRA, M.E.B.; BORGES, M.F.; NASSU, R.T.; RODRIGUES, C.L.J.; ALMEIDA, G.B. de. Polpa de acerola: avaliação química, físico-química, microbiológica e sensorial durante o armazenamento. In: SIMPÓSIO LATINO AMERICANO DE CIÊNCIA DE ALIMENTOS, 4., Campinas, SP. Resumos... Campinas: Editora da Universidade Estadual de Campinas, 2001. p. 274.

28 SILVA, F. A. S.; AZEVEDO, C. A. V. A new version of the ASSISTAT - Statistical Assistance Software. In: WORLD CONGRESS ON COMPUTERS IN AGRICULTURE, $4^{\text {th }}$, Orlando, 2006. Proceedings... Orlando, FL: American Society of Agricultural Engineers, 2006. p. 393-396.

29 SILVA, M.F.V.; MENEZES, H.C.; GUEDES, M.C. Efeito de diferentes tratamentos térmicos sobre as antocianinas na polpa de acerola. In: SIMPÓSIO LATINO AMERICANO DE CIÊNCIA DE ALIMENTOS, 3., Campinas. Anais... Campinas: UNICAMP, 1999. p. 152-153.

30 VENDRAMINI, A.L.; TRUGO, L.C. Chemical composition of acerola fruit (Malpighia punicifolia L.) at three stages of maturity. Food Chemistry, v. 71, p. 195-198, 2000.

31 VICENTE, A.M.; RUBIO, J.G.P.; REGIDOR, F.S. Refrigeración, congelación y envasado de los alimentos. Madri: AMV, Ediciones Mundi Prensa, 1994. 276 p.

32 YAMASHITA, F.; BENASSI, M. T.; TONZAR, A. C.; MORIYA, S.; FERNANDES, J. G. Produtos de acerola: estudo da estabilidade de vitamina C. Ciência e Tecnologia de Alimentos, v. 23, n.1, p. 92-94, 2003.

\section{AGRADECIMENTOS}

A primeira autora agradece a FAPITEC (SE) pela concessão da bolsa de mestrado. 